International Research Journal of Diabetes and Metabolism (DOI:10.28933/IRJDM)

\title{
The knowledge of users with diabetes mellitus of a basic health unit on diabetic foot
}

\section{Silva E.C.S1, Nascimento J.W.A2, Siqueira A.C.P.R3, Jesus S.B4}

1,2Nursing Student In the Católica of Pernambuco University; 3Medical Student In Católica of Pernambuco University; 4Docent/ researcher in the Católica of Pernambuco University.

\begin{abstract}
Introduction: The Diabetic foot is a neuropathy responsible for several alterations and complications, particularly in the lower limbs. The way diabetics behave and how well they know about diabetic foot care is of ultmost importance, considering prior knowledge precedes an effective preventive plan. Objective: This study aims to investigate the knowledge of diabetic pacients, who are users of the Basic Health Unit in the city of Olinda, on the diabetic foot. Methodology: This is an exploratory descriptive study of quantitative methods, with a sample of 50 diabetic patients from BHU users. After signing a Free and Informed Consent Form, a questionnaire was applied and the collected data was consolidated and presented in tables and graphs charts. Results and Discussion: In total, $42 \%$ of diabetics users of the BHU reported that they had not heard about diabetic foot, while $93 \%$ reported that they knew about the risks of foot amputation; about $88 \%$ affirmed to take care of their feet and the main precautions mentioned were: washing their feet (54\%), avoiding to walk barefoot (39 \%) wipe the feet properly (32\%), 81\% didn't know about risk factors and approximately $69 \%$ reported that they received no professional guidance in the basic health unit regarding foot care. Conclusion: In light of the above, it is noticeable the importance of a greater knowledge of diabetics users on the neuropathic foot and the need for specific care. Therefore, the understanding is essential for prevention of complications, such as amputation of lower limbs, and for decreased morbimortality.
\end{abstract}

Keywords: Knowledge; Diabetes mellitus; Diabetic foot
*Correspondence to Author:

Silva E.C.S

Nursing Student In the Católica of

Pernambuco University

How to cite this article:

Silva E.C.S, Nascimento J.W.A, Siqueira A.C.P.R, Jesus S.B.The knowledge of users with diabetes mellitus of a basic health unit on diabetic foot.International Research Journal of Diabetes and Metabolism, 2018, 1:2

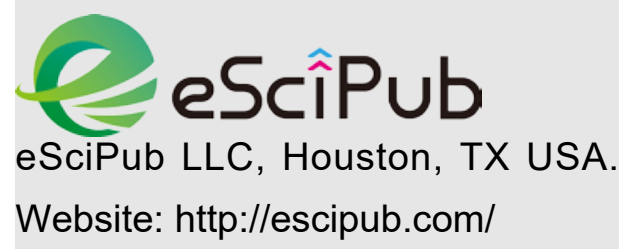

